\title{
Apuntes sobre el arbitraje en China y el reconocimiento de laudos ${ }^{(*)}$
}

\section{Notes on arbitration in China and recognition of awards}

\author{
José Tam Pérez ${ }^{(*)}$ \\ Universidad de Lima \\ Claudia Martinez Zúñiga ${ }^{(* *)}$ \\ Universidad Católica de Santa María de Arequipa
}

\begin{abstract}
Resumen: El presente trabajo versa sobre el arbitraje en China y el reconocimiento de los laudos arbitrales extranjeros en China y Perú. El autor indica cómo era el arbitraje y la solución de controversias en China, también señala que la apertura comercial china generó que acoja dentro de su ordenamiento jurídico al arbitraje comercial. Sobre esa línea, señala que el principal instrumento sobre el reconocimiento y ejecución de los laudos extranjeros en China y Perú es la Convención de Nueva York. Sobre el procedimiento del reconocimiento y ejecución de los laudos extranjeros se dice cómo se realiza en el derecho chino y en el peruano. El autor añade que China y Perú deberían tener un proceso de conocimiento mutuo de solución de controversias para asegurar las inversiones mutuas.
\end{abstract}

Palabras Claves: Arbitraje en China - Reconocimiento de Laudos Arbitrales Extranjeros - Convención de Nueva York - Solución de Controversias Ejecución de Laudos Arbitrales Extranjeros - Arbitraje en Perú

Abstract: This paper is about the arbitration in China ant he recognition of the foreign arbitral awards in China and Peru. The author indicates how the

${ }^{*}$ ) Nota del Editor: El artículo fue recibido el 15 de noviembre de 2017 y aprobada su publicación el 30 de noviembre de 2017.

$\left(^{* *}\right.$ Abogado por la Universidad de Lima. Estudios de Doctorado en Derecho Procesal en la Universidad Complutense de Madrid. Especialista en Litigios y arbitraje. Socio a cargo del área de litigios y arbitraje en Rodrigo, Elías y Medrano. Socio a cargo del área de inversiones asiáticas. Actualmente preside la Cámara de Comercio Peruano China. Correo electrónico: jtam@ estudiorodrigo.com

${ }^{(* *}$ ) Abogada por la Universidad Católica de Santa María de Arequipa. Maestría en Inversiones y Cooperación Internacional China por la University of International Business and Economics. Maestría en Derecho y Globalización por Maastricht University. Estudios generales de Derecho Chino para extranjeros por la University of Political Sciences and Law. Nombrada joven sinóloga por el Gobierno de China. Asociada senior del área Corporativa y Comercial y del área de Inversiones Asiáticas. Correo electrónico: cmartinez@estudiorodrigo.com 


\section{Apuntes sobre el arbitraje en China y el reconocimiento de laudos Notes on arbitration in China and recognition of awards}

arbitration and the dispute settlement were in China, also he says that the Chinese commercial opening produced that China adopted inside its legal system the commercial arbitration. Above this line, he says that the main instrument regarding the recognition and enforcement of the foreign arbitral awards in China and Peru is the New York Convention. About the proceeding of this recognition and enforcement of the foreign arbitral awards it is said how it is performed in the Chinese and Peruvian Law. The author ads that China and Peru should have a mutual awareness proceeding of the dispute settlement to ensure the mutual investments.

Keywords: Arbitration in China - Recognition of the Foreign Arbitral Awards - New York Convention - Dispute Settlement Enforcement of the Foreign Arbitral Awards - Arbitration in Peru

Sumario: 1. Introducción_2. Arbitraje y solución de controversias en China_3. Instrumentos normativos que rigen el reconocimiento y ejecución de laudos arbitrales extranjeros en China y en Perú_4. Procedimiento de reconocimiento y ejecución de laudos arbitrales extranjeros en China y Perú

\section{Introducción}

El primer rastro de relación comercial entre Perú y China lo encontramos en el siglo XVI cuando barcos españoles cargados con plata de Perú y México llegaban a las costas de Manila (Filipinas), donde ávidos comerciantes chinos esperaban adquirir el preciado mineral y, a su vez, vender su mercadería (seda y porcelana principalmente). Posteriormente, a partir del siglo $\mathrm{XIX}$, las relaciones comerciales se consolidarían con la llegada de los primeros ciudadanos chinos al Perú quienes dieron inicio a un proceso de inmigración asiática sin precedentes en la región.

Actualmente, China se ha convertido en el principal socio comercial del Perú como consecuencia de una política de
Estado que se inicia en 1992, año en el que se realizó la primera inversión directa china en el sector minero(1). Desde entonces, cada gobierno ha seguido desarrollando y fortaleciendo las relaciones bilaterales, Io que nos ha permitido ser uno de los pocos países en la región en tener un Tratado de Libre Comercio con China ${ }^{(2)}$ y una Asociación Estratégica Integral(3).

En este contexto, las relaciones comerciales y de inversión entre China y Perú se encuentran en pleno desarrollo, no solo porque China es el principal socio comercial del Perú, luego de desplazar a Estados Unidos; sino, porque el nuevo contexto de alianzas comerciales internacionales va a dinamizar aún más esta relación. Ello, considerando el apartamiento de Estados Unidos del Tratado Transpacífico (En adelante, "TPP") y la importancia que tomará la Asociación Económica Integral Regional (En adelante, "RCEP" por sus siglas en inglés) impulsada por China, además del proyecto de creación de una zona de libre comercio del Asía Pacífico que supone la interacción económica entre las 21 economías miembros del Foro, que representan el $60 \%$ del PBI mundial y el $50 \%$ del comercio mundial.

En el marco descrito, es de esperarse que el crecimiento en las relaciones comerciales entre ambos países suponga un aumento en la celebración de contratos entre partes peruanas y chinas y, como consecuencia de ello, un aumento en el número de controversias. Dado el rol central que ocupa

(1) El 5 de noviembre de 1992, Shougang Corporation, empresa estatal china dedicada a la producción de hierro, acero y otros minerales, adquirió Hierro Perú. Más información puede ser encontrada en: Congreso Perú, "La privatización del empresa Shougang Hierro Perú S.A.A.," http://www4.congreso.gob.pe/comisiones/2002/CIDEF/resumenes/privatiza/hierro. pdf (consultada el 06 de abril de 2017).

(2) El Tratado de Libre Comercio entre el Gobierno de la de República del Perú y el Gobierno de la República Popular de China, fue suscrito el 28 de abril de 2009 y ratificado mediante Decreto Supremo No. 092-2009-RE publicado en el Diario Oficial El Peruano el 6 de diciembre de 2009.

(3) La Asociación Estratégica Integral entre el Gobierno del Perú y la República Popular de China fue suscrita el 6 de abril del 2013. 
José Tam Pérez y

Claudia Martinez Zúñiga

el arbitraje internacional en la solución de controversias comerciales internacionales; no debe resultar extraño que presenciemos un aumento en el número de arbitrajes internacionales entre partes peruanas y chinas. Así, es fundamental que los abogados, centros de arbitraje, árbitros y autoridades jurisdiccionales peruanas cuenten con información básica para el manejo de este tipo de controversias.

\section{Arbitraje y solución de controversias en China}

Un primer punto a tratar es el referido al particular entendimiento del conflicto en la cultura china. Los conceptos de armonía y cooperación son fundamentales en China, pues forman parte intrínseca de su cultura. Una disputa se considera un mal que perturba la armonía que gobierna toda la vida social, por ende, existe un desdén hacia el litigio. En la cultura china se considera que si es posible evitar una disputa que vaya a afectar las relaciones a largo plazo, es imperativo que las partes (ya sea por sí mismas o con la ayuda de un tercero) tomen las medidas necesarias para eliminar amigablemente las causas del problema.

El confucianismo, con su énfasis en la armonía (o He Wei Gui), es la base conceptual para el predominio de la mediación en China como método de resolución de conflictos debido a que los principales valores confucianos: la benevolencia (Ren), la lealtad (Yi), el ritualismo (Li), la sabiduría (Zhi) y la sinceridad (Xin), promueven que las personas busquen esa armonía social, evitando conflictos y buscando solucionar disputas a través de la persuasión moral y no por la fuerza de la ley (Yao Xinzhong 2000, 81-96 y 170-78). Una de las principales consecuencias de no evitar el conflicto era y es el perder la cara (mianzi) ${ }^{(4)}$. Este concepto, que aún se mantiene muy arraigado en la cultura china, genera, entre otros, que los demás miembros de la comunidad pierdan la confianza y sus negociaciones futuras se vean perjudicadas, pues no es respetable hacer negocios con personas que provoquen conflictos o no traten de evitarlos. El confucianismo busca que las personas internalicen estos valores y las reglas del deber para con la sociedad, a fin de preservar la armonía y cooperación.

En ese sentido, no sorprende que el método de resolución de conflictos que se ha venido utilizando con más frecuencia en China sea la mediación (Kaufmann-Kohler y Kun 2008). Hay registros que prueban que la mediación fue utilizada durante la dinastía Zhou Occidental (1146 aC - $771 \mathrm{aC}$ ) y luego, a nivel nacional, durante la dinastía Qin (221 aC - 207 aC) hasta Qing (última dinastía) (Sun 2011). Incluso luego, con el establecimiento del legalismo como corriente contraria al confucianismo y por ende el establecimiento del Estado de Derecho durante el siglo III a.C., la preferencia por utilizar la mediación como método de resolución de conflictos se mantuvo (Kun 2013, 163-64).

La posterior apertura comercial de China al mundo generó también que acoja dentro de su ordenamiento jurídico al arbitraje comercial. Aunque el arbitraje en China se viene aplicando desde 1950 a través de la Comisión Internacional de Arbitraje Económico y Comercial de China (En adelante, "CIETAC"), fue recién en 1995 cuando entró en vigor la ley de Arbitraje de China, que oficialmente reguló el arbitraje como medio alternativo de resolución de conflictos. El moderno sistema arbitral, se constituyó sobre la bases de distintas comisiones arbitrales que resolvían controversias de carácter comercial y administrativo.

Se diferenciaba entre controversias domésticas e internacionales. Únicamente, la Comisión de Arbitraje Marítimo de China (En adelante,

(4) Cónfer M. CHAN, Alvin The Chinese Concepts of Guangxi, Mianzi, Renqing and Bao: Their Interrelationship and Implications for International Business, University of Western Sydney, 2006. Una versión online puede encontrarse en: Australian \& New Zeland Marketing Academy http://www.anzmac.org/conference_archive/2006/documents/Chan_Alvin.pdf (consultada el 06 de abril de 2017). 


\section{Apuntes sobre el arbitraje en China y el reconocimiento de laudos Notes on arbitration in China and recognition of awards}

"CMAC")(5) y la Comisión Internacional de Arbitraje Económico y Comercial de China ${ }^{(6)}$ podían administrar arbitrajes que involucrasen partes extranjeras. Además de su sede en Beijing, CIETAC contaba con subcomisiones en Shangai y en la zona económica especial de Shenzen. De acuerdo con la nota interpretativa de la Corte Suprema de Justicia de China del 15 de julio de 2015, estas comisiones obtuvieron independencia de CIETAC.

Por mandato de la misma nota interpretativa, y para evitar conflictos de jurisdicción entre CIETAC Beijing y las subcomisiones en Shenzhen y Shanghai, estas últimas pasaron a denominarse Comisión del Sur de China en Arbitraje Comercial y Económico Internacional, también conocida como Corte Internacional de Arbitraje Shenzhen (En adelante, "SCIA"), y Comisión de Arbitraje Económico y Comercio Internacional de Shanghai, también conocida como Centro de Arbitraje de Shanghai (En adelante, "SHIAC"). Esta división de la CIETAC originó tres instituciones independientes de arbitraje en las tres ciudades más dinámicas de China.

Con la entrada en vigencia de la Ley de Arbitraje de China ${ }^{(7)}$, se adoptaron diversos estándares propios del arbitraje internacional. En resumen, la norma arbitral dispone que (i) las partes son libres de decidir que su controversia sea resuelta a través del arbitraje $^{(8)}$; (ii) las partes tienen derecho a decidir quiénes resolverán la controversia y dónde se llevará a cabo el procedimiento(9); (iii) el arbitraje es independiente del poder estatal(10); y, (iv) los laudos son finales y de obligatorio cumplimiento para las partes.

De igual modo, es importante señalar que China es parte de una serie de acuerdos en materia de inversión extranjera ${ }^{(11)}$ y en materia de libre comercio con diversos Estados. Muchos de estos acuerdos incluyen la aceptación del Estado de resolver las controversias con inversionistas extranjeros a través del arbitraje. Cabe mencionar, en este marco, que el primer arbitraje de inversiones - al amparo de las disposiciones del APPRI Perú-China ${ }^{(12)}$ - que involucró un ciudadano chino fue contra Perú (caso Tza Yap Shum c. Perú).

(5) La Comisión de Arbitraje Marítimo de China establecida por el Consejo Chino para la Promoción del Comercio Internacional (En adelante, "CCPIT") el 22 de enero de 1959, está a cargo de la administración de casos nacionales e internacionales que involucran disputas marítimas. La CMAC tiene su sede en Beijing, con sub-comisiones en Shanghai, Tianjin, Chongqing, Shenzhen, Hong Kong y Fujian. Los laudos de la CMAC se consideran imparciales y han ganado gran reputación en el país y en el extranjero. La CMAC ahora es una de las principales instituciones de arbitraje marítimo.

(6) La Comisión Internacional de Arbitraje Económico y Comercial de China es una de las principales instituciones de arbitraje permanente en el mundo. Anteriormente era conocida como la Comisión de Arbitraje de Comercio Exterior. CIETAC se creó en Abril de 1956 y en 1988 adquirió su actual denominación. Desde el año 2000, CIETAC también es conocida como la Corte de Arbitraje de la Cámara de Comercio Internacional de China (En adelante, "CCOIC").

(7) The Arbitration Law of the People's Republic of China, promulgada el 31 de Agosto de 1994. Una versión online de la misma puede ser consultada en: www.wipo.int/wipolex/en/text.jsp?file_id=182634 (consultada el 06 de abril de 2017).

(8) El artículo 4 de la Ley de Arbitraje china señala: "La remisión a arbitraje que las partes hagan para resolver sus disputas mediante arbitraje debe efectuarse sobre la base del libre consentimiento de ambas partes y del acuerdo arbitral por éstas celebrado. Si una parte solicita el arbitraje en ausencia de acuerdo arbitral, la comisión de arbitral debe rechazar el caso" (Traducción libre del autor).

(9) El segundo párrafo del artículo 16 de la Ley de Arbitraje china dispone: "El acuerdo arbitral debe contener lo siguiente: (1) la expresión de la intención de acudir al arbitraje; (2) los asuntos relativas al procedimiento arbitral; y (3) el nombre de la comisión de arbitraje designada para administrar el procedimiento" (traducción libre del autor).

(10) En particular, el artículo 14 de la Ley de Arbitraje china dispone: "Las comisiones de arbitraje deben ser independientes de los órganos administrativos y no deben existir relaciones de subordinación entre las comisiones arbitrales y los órganos administrativos (...)" (Traducción libre del autor).

(11) Acuerdos más conocidos como Tratados Bilaterales de Inversión (En adelante, “TBI").

(12) Acuerdo entre el Gobierno de la República del Perú y el Gobierno de la República Popular China sobre Promoción y Protección Recíproca de Inversiones (o también, TBI Perú-China), ratificado el 01 de febrero de 1995. Una versión online puede ser consultada en: http://investmentpolicyhub.unctad.org/Download/TreatyFile/768 (consultada el 06 de abril de 2017). 
José Tam Pérez y

Claudia Martinez Zúñiga

Finalmente, una mención aparte merece la regulación arbitral en Hong Kong siendo que las consideraciones antes expuestas aplican únicamente a China continental. El arbitraje está regulado en Hong Kong mediante su propia Ley de Arbitraje ${ }^{(13)}$, que, entre otras cosas, se diferencia de la Ley de Arbitraje china en su aproximación monista al arbitraje internacional; no distinguiendo entre arbitrajes domésticos e internacionales ${ }^{(14)}$. Además, debe señalarse que es común que partes chinas recurran al Hong Kong International Arbitration Center (En adelante, "HKIAC") para resolver sus controversias, como al arbitraje con sede en Hong Kong bajo las reglas de la Corte Internacional Arbitraje de la Cámara de Comercio Internacional (En adelante, "CCl").

\section{Instrumentos normativos que rigen el reconocimiento y ejecución de laudos arbitrales extranjeros en China y en Perú}

El principal instrumento al que habrá que remitirse a efectos de determinar el procedimiento de reconocimiento y ejecución de laudos arbitrales extranjeros en China y Perú es la Convención de Nueva York para el reconocimiento y ejecución de sentencias arbitrales extranjeras de 1958 (En adelante, "Convención de Nueva York"). Así, de conformidad con el artículo 28 del Tratado de Asistencia Judicial en Materia Civil y Comercial entre Perú y China(15), los laudos arbitrales serán reconocidos de conformidad con la Convención de Nueva York. La remisión a dicho instrumento está también dispuesta en el artículo 269 del Código Procesal Civil Chino, que regula la competencia judicial para conocer el reconocimiento y ejecución la aplicación de los tratados internacionales de los que China es parte.

En primer lugar, al analizar la aplicación de la Convención de Nueva York en un caso concreto, es relevante tener en cuenta que de acuerdo al derecho chino pueden distinguirse hasta tres tipos de arbitraje: (i)los arbitrajes domésticos (aquellos dictados en China por alguna comisión de arbitraje chino sin elemento extranjero alguno), (ii) los arbitrajes con algún elemento extranjero (esto es, arbitrajes administrados por comisiones arbitrales en China con algún elemento extranjero); $y$, (iii) los arbitrajes extranjeros (esto es, arbitrajes dictados fuera de China) (D. Friedland y Yan 2011, 468).

Aquellos arbitrajes de la segunda categoría, arbitrajes con algún elemento extranjero, le serán aplicables el artículo 260 del Código Procesal Chino que permite denegar el reconocimiento y ejecución del laudo bajo causales similares a las recogidas en la Convención de Nueva York. Sin embargo, dicha norma también permite denegar el reconocimiento y ejecución cuando ello vulnere el interés público y social del Estado (China) ${ }^{(16)}$.

En segundo lugar debe mencionarse que, tal como lo permite la Convención de Nueva York, las partes pueden efectuar reservas a su aplicación. La Convención permite que los Estados se obliguen únicamente a reconocer laudos al amparo de la Convención de Nueva York siempre que (i) deriven de relaciones jurídicas consideradas como comerciales de conformidad con su derecho interno; y (ii) hayan sido dictados en Estados que son parte de la Convención.

(13) Arbitration Ordinance (Capítulo 609) en vigencia desde el 01 de junio de 2011. Una versión online de la misma puede ser consultada en: http://www.wipo.int/edocs/lexdocs/laws/en/hk/hk185en.pdf (consultada el 06 de abril de 2017).

(14) Al igual que en el caso peruano, la Ley de Arbitraje de Hong Kong reproduce casi textualmente las disposiciones de la Ley Modelo de Arbitraje Internacional de la Comisión de Naciones Unidas para la Unificación del Derecho Mercantil Internacional (CNUDMI).

(15) El tratado se encuentra en vigencia desde el 25 de mayo de 2012 y regula, entre otros aspectos, la notificación de resoluciones judiciales, actuación y obtención de pruebas, reconocimiento y ejecución de laudos arbitrales, e intercambio de información y jurisprudencia.

(16) Así, el artículo 258 del Código Procesal Chino establece que: "Si la Corte determina que la ejecución del laudo violara el interés público y social, la Corte debe rechazar la ejecución del laudo" (Traducción libre del autor). 


\section{Apuntes sobre el arbitraje en China y el reconocimiento de laudos Notes on arbitration in China and recognition of awards}

China ha adoptado ambas reservas ${ }^{(17)}$, mientras Perú no ha adoptado ninguna.

En tercer lugar, hay que apuntar que la Convención de Nueva York recoge causales taxativas para denegar el reconocimiento y ejecución de laudos arbitrales extranjeros. En el caso peruano, la Ley de Arbitraje ha reproducido casi textualmente dichas causales. Sobre las particularidades en la aplicación de las mismas cabe resaltar que:

a) La no arbitrabilidad de la materia sometida a arbitraje, que constituye una causal de denegación del reconocimiento y ejecución de laudos extranjeros debe ser analizada en el caso chino en concordancia con los artículos 2 y 3 de la Ley de Arbitraje China. En particular el artículo 3, dispone que dentro de las disputas que no pueden someterse a arbitraje estén aquellas de carácter administrativo que por mandato legal sean de competencia de los órganos administrativos estatales. Por su parte, en el caso peruano la no arbitrabilidad debe leerse a partir del artículo 2 que dispone que solo pueden someterse a arbitraje las controversias sobre materia de libre disposición;

b) En relación a la causal de orden público, que suele ser usada como cajón de sastre para oponerse al reconocimiento y la ejecución de laudos extranjeros, la práctica judicial china ha tendido a la interpretación restrictiva del orden público en una clara manifestación favorable al arbitraje internacional(18). Por su parte, la Ley de Arbitraje peruana señala que el orden público es el orden público internacional delimitando considerablemente el debate que suele surgir en torno a la Convención de Nueva York. Esta delimitación es significativa pues el artículo 75 de la Ley de Arbitraje peruana señala que en casos las disposiciones recogidas en la misma sean más favorables al reconocimiento y ejecución del laudo extranjero, éstas deben primar por sobre las de la Convención de Nueva York. En consecuencia, al contener la Convención de Nueva York una causal más restrictiva al reconocimiento y ejecución ${ }^{(19)}$, será de aplicación la causal de orden pública recogida en la Ley de Arbitraje.

Finalmente, de estar frente a un laudo emitido contra China o que involucra a un inversionista chino en un arbitraje de inversiones, dependerá del tipo de arbitraje frente al que nos encontremos (teniendo en cuenta la calificación antes expuesta) para determinar si la Convención de Nueva York es aplicable al eventual reconocimiento y/o ejecución. De estar frente a un arbitraje bajo las reglas del Convenio del Centro Internacional de Arreglo de Diferencias

(17) En relación a la reserva de comercialidad, la nota interpretativa emitida por la Corte Suprema ha delimitado exhaustivamente los supuestos de en los que se configura una relación jurídica de carácter comercial. Cónfer Circular of Supreme People's Court on Implementing Convention on the Recognition and Enforcement of Foreign Arbitral Awards Entered by China, una versión online puede ser consultada en: $\mathrm{http}: / / \mathrm{www}$.cietac.org/index.php?m=Article\&a=show\&id=2413\&l=en (consultada el 06 de abril de 2017).

(18) El ex juez de la Corte Suprema China, Lu Xialong, se refería a esta actitud china hacia el arbitraje internacional en los siguientes términos: "[L]as Cortes chinas han adoptado siempre gran importancia al examen de laudos extranjeros, cuyo reconocimiento y ejecución debe ser decidido estrictamente de conformidad con la Convención de Nueva York y el derecho chino (...) han adoptado [igualmente] una política activa y de apoyo con respecto al arbitraje doméstico e internacional fundada en el derecho". Cónfer Xialong, Lu. "The Recognition and Enforcement of Foreign Arbitral Awards in China," en New Horizons in the International Commercial Arbitration and Beyond, Van den Verg. Kluwer Arbitration International, 2007, 347.

(19) La Convención de Nueva York dispone que "Que el reconocimiento o la ejecución de la sentencia serían contrarios al orden público de ese país." Esto ha dado lugar a copiosa jurisprudencia interpretando dicha referencia al orden público como referente al orden público interno (del Estado donde se pide el reconocimiento y ejecución), de modo que la Ley Peruana al adoptar acertadamente la referencia al orden público internacional previene la denegación de reconocimiento y ejecución por causales domésticas. 
José Tam Pérez y

Claudia Martinez Zúñiga

Relativas a Inversiones (En adelante, "CIADI"), por mandato del artículo 54 de dicho instrumento, los laudos CIADI se ejecutan como si se tratase de sentencias firmes emitidas en el Estado donde se solicita el reconocimiento y/o ejecución. Caso distinto será el de arbitrajes de inversiones tramitados bajo el Reglamento de Arbitraje de la Comisión de Naciones Unidas para la Unificación del Derecho Mercantil Internacional (En adelante, "CNUDMI") u otras reglas de arbitraje, pues en aquellos casos, al no haber una disposición como la citada, los laudos serán tratados como laudos extranjeros y por ende deberán pasar para su reconocimiento y/o ejecución por los filtros de la Convención de Nueva York.

\section{Procedimiento de reconocimiento y ejecución de laudos arbitrales extranjeros en China y Perú}

La Ley de Arbitraje china remite a las disposiciones del Código Procesal Civil Chino en materia de reconocimiento y ejecución de laudos y sentencias extranjeras a fin de determinar cuál es el procedimiento a seguirse. En el caso peruano, la Ley de Arbitraje contiene un título específico que recoge las normas relativas al reconocimiento y ejecución de laudos arbitrales extranjeros ${ }^{(20)}$. Aun cuando no lo menciona expresamente, debe entenderse que para todo aquello que no esté regulado en relación al proceso judicial de reconocimiento y ejecución de laudos extranjeros, son de aplicación las normas del Código Procesal Civil peruano.

Para los procesos de reconocimiento y ejecución, la legislación arbitral China dispone, a partir de una nota interpretativa emitida por la Corte Suprema China en abril de 2000, un mecanismo de apelación automática. Así, la parte que demande el reconocimiento y ejecución debe recurrir a un Juzgado (Intermediate People' High Court) $^{(21)}$. Si el Juzgado determina que se han configurado alguna de las causales de denegatoria del reconocimiento y ejecución de la Convención de Nueva York, entonces deberá remitir el asunto a conocimiento de la Corte Superior (High People'Court). Si dicha Corte rechaza el reconocimiento y ejecución deberá remitir el asunto a la Corte Suprema. Únicamente recibido el dictamen de dicha Corte, el Juzgado podrá denegar el reconocimiento y ejecución ${ }^{(22)}$. Esto ha permitido una mejora en la práctica judicial china en la materia, especialmente al aplicar causales de denegatoria de reconocimiento y ejecución cuyo contenido es controversial, como el orden público. En el caso peruano, este mecanismo de apelación de oficio no está contemplado, sin embargo debe señalarse que por mandato del articulo 76.2 de la Ley de Arbitraje peruana, la corte competente es una Corte Superior y contra lo decidido solo procede recurso de casación.

En el caso chino, el Juez competente para conocer el pedido de reconocimiento y ejecución será el juez del domicilio del demandado en caso se trate de una persona natural y el juez de la localidad de la sede principal en caso de trate de una persona jurídica; en caso el demandado no tenga domicilio o sede principal en China, el competente será el juez del lugar donde se encuentran sus activos ${ }^{(23)}$. El mismo criterio lo encontramos reproducido para el caso peruano, que para tal efecto se remite a las normas generales de la competencia recogidas en el Código Procesal Civil.

(20) Título VIII, Reconocimiento y Ejecución de Laudos Extranjeros, Decreto Legislativo No. 1071.

(21) Estos Juzgados deberán ser, por competencia territorial, los de ciudades capitales de provincias y los de ciudades capitales de regiones autónomas y municipalidades directamente bajo jurisdicción del Gobierno Central. Cónfer Provisions of the Supreme People's Court on Some Issues Concerning the Jurisdiction of Civil and Commercial Cases Involving Foreign Elements, 03 de enero de 2002.

(22) Véase Circular of the Supreme People's Court on Issues in the People's Courts Handling of Foreign-related Arbitrations and Foreign Arbitrations, 28 de agosto de 1995, una versión online puede ser consultada en: http://www.cietac.org/index. php?m=Article\&a=show\&id=2414\&l=en (consultada el 06 de abril de 2017).

(23) Véase Circular of Supreme People's Court on Implementing Convention on the Recognition and Enforcement of Foreign Arbitral Awards Entered by China, una versión online puede ser consultada en: http://www.cietac.org/index. php?m=Article\&a=show\&id=2413\&l=en (consultada el 06 de abril de 2017). 


\section{Apuntes sobre el arbitraje en China y el reconocimiento de laudos Notes on arbitration in China and recognition of awards}

En relación a los requisitos formales de la demanda, ambos países han acogido las disposiciones de la Convención de Nueva York. De conformidad con estas reglas, la parte solicitante debe presentar el laudo original debidamente autenticado así como el original del convenio arbitral. Adicionalmente, si el laudo no estuviese en el idioma del Estado donde se solicita el reconocimiento y ejecución, debe ser acompañado de una traducción al idioma local; dicha traducción debe ser certificada por un traductor oficial.

En el caso chino, mediante nota interpretativa de la Corte Suprema, se ha establecido con claridad que con la solicitud de reconocimiento y/o ejecución del laudo extranjero deben cumplirse los siguientes requisitos: (i) Expresar en la solicitud las razones por las que se solicita la ejecución, de ser el caso, ya sea por escrito u oralmente; (ii) Acompañar la solicitud con la traducción certificada por autoridad consular china o notario chino.

Finalmente, debe apuntarse que a efectos de asistencia judicial durante el proceso de reconocimiento y/o ejecución de laudos extranjeros se estará a las disposiciones del ya citado Tratado de Asistencia Judicial en Materia Civil y Comercial entre Perú y China. De conformidad con este instrumento, tanto los tribunales chinos como los peruanos pueden solicitar mutuamente el envío de documentos judiciales, investigación, obtención de pruebas de pruebas $u$ otras acciones judiciales. El procedimiento, conforme se encuentra detallado, impone la obligación de los Estados de enviar una carta de solicitud de asistencia judicial y anexos al tribunal correspondiente, adjuntando para ello traducciones al chino o al castellano dependiendo del caso(24).
De la mano del desarrollo de las relaciones comerciales y de inversión, China y Perú (Latinoamérica), deberán iniciar también un proceso de conocimiento mutuo en el ámbito jurídico y, especialmente, en la cultura de solución de controversias. Será un proceso no exento de dificultades que ambos países deberán ir superando, con el fin de dar seguridad a las inversiones mutuas. En este contexto, abogados, centros de arbitraje, árbitros y órganos jurisdiccionales tendremos el reto de adaptarnos a los conflictos asiático - latinoamericanos y sobresalir como país en la región, de la manera en la que lo hemos estado haciendo hasta hoy en nuestra relación bilateral con China.

\section{Referencias bibliográficas}

D. Friedland y Bing Yan. 2011. Negotiating and Drafting Arbitration Agreements with Chinese Parties. Special Considerations Paul of Chinese Law and Practice, en Journal of International Arbitration, vol. 28, No. 5: 467-484.

Kaufmann-Kohler, Gabrielle y Fan Kun. 2008. Integrating Mediation into Arbitration: Why It Works in China. Journal of International Arbitration, vol. 25, No. 4: 479-492.

Kun Fan. 2013. Arbitration in China: A Legal and Cultural Analysis. Bloomsbury Publishing.

M. Chan, Alvin. 2006. The Chinese Concepts of Guangxi, Mianzi, Renging and Boo: Their Interrelationship and Implications for Internacional Business. University of Western Sydney.

孙巍(Sun Wei). 2011.中国商事仲裁法律与实务 (Law and Practice of Chinese Commercial Arbitration), 北京大学出版社 (Beijing University).

Xialong, Lu. 2007. The Recognition and Enforcement of Foreign Arbitral Awards in China, en New Horizons in the International Commercial Arbitration and Beyond, 346-349. Van den Berg, Jan, (ed.). Kluwer Arbitration International.

Yao Xinzhong. 2000. An Introduction to Confucianism. Cambridge University Press. https://doi.org/10.1017/ cbo9780511800887 R[|6

(24) Uno de los mayores problemas que se presente actualmente en el reconocimiento y ejecución de laudos chinos en el Perú es que sólo se cuenta con tres traductores públicos de chino mandarín para cubrir una demanda cada vez más creciente. 